\title{
Influence of molecular dissociation on blob-induced atom density perturbations Paper
}

Thrysøe, A. S.; Madsen, J.; Naulin, V.; Juul Rasmussen, J.

\section{Published in:}

Nuclear Fusion

Link to article, DOI:

10.1088/1741-4326/aac8a4

Publication date:

2018

Document Version

Peer reviewed version

Link back to DTU Orbit

Citation (APA):

Thrysøe, A. S., Madsen, J., Naulin, V., \& Juul Rasmussen, J. (2018). Influence of molecular dissociation on blob-induced atom density perturbations: Paper. Nuclear Fusion, 58(9), [096005]. https://doi.org/10.1088/17414326/aac8a4

\section{General rights}

Copyright and moral rights for the publications made accessible in the public portal are retained by the authors and/or other copyright owners and it is a condition of accessing publications that users recognise and abide by the legal requirements associated with these rights.

- Users may download and print one copy of any publication from the public portal for the purpose of private study or research.

- You may not further distribute the material or use it for any profit-making activity or commercial gain

- You may freely distribute the URL identifying the publication in the public portal

If you believe that this document breaches copyright please contact us providing details, and we will remove access to the work immediately and investigate your claim. 


\title{
Influence of molecular dissociation on blob-induced atom density perturbations
}

\author{
A.S. Thrysøe, J. Madsen, V. Naulin, and J. Juul Rasmussen \\ PPFE, Department of Physics, DTU, DK-2800 Kgs, Lyngby, Denmark \\ E-mail: alec@fysik.dtu.dk
}

16 May 2018

\begin{abstract}
The effects of enhanced electron and ion pressure perturbations mediated in filamentary structures (blobs) on the densities of neutral atoms and molecules are investigated through a self-consistent dynamical fluid model for plasma and neutral fields. The electron and ion densities and pressures, and the generalized vorticity, are simulated by a 2D drift-fluid model in an edge and scrape-off layer slab domain of a toroidally magnetically confined plasma. The plasma dynamics are coupled with a diffusion model for densities of neutral atoms and molecules. The combined model allows for determining the response of the density of neutrals with various temperatures to blobs. It is found that blobs locally deplete densities of molecules and atoms that do not originate from dissociation of molecules, whereas the density of atoms created by dissociation may increase during blob events. The neutral species, their temperature, and origin should thus be taken into consideration when estimating the effect of blobs on neutral density perturbations when calculating emission rates, e.g., for gas puff imaging.
\end{abstract}

\section{Introduction}

The conditions in the edge and scrape-off layer (SOL) regions of magnetically confined plasmas contribute significantly to determining the overall confinement properties, as they function as boundary conditions for the confined plasma [1]. The edge and SOL regions are separated by the last-closed-flux-surface (LCFS), across which the outwards transport of particles and energy is known to be highly intermittent. In the SOL the plasma is mainly transported in field-aligned structures of enhanced electron and ion 
pressures, known as blobs, which are formed near the LCFS and propagate radially outwards [2, 3].

The spatial structure of blobs can be observed using the gas puff imaging (GPI) technique [4, 5, 6]. Neutral gas, for example hydrogen isotopes or helium, is puffed into the SOL, and the electrons from the blob excite the atoms, which radiate in the visible spectrum. Fast cameras monitor the location and magnitude of radiation, and the data allows for deriving information about the magnitude and location of the electron density and temperature fields. In the GPI calculations it is common to assume a static background of neutral particles. However, in recent papers [7, 8, 9] this assumption has been questioned, and simulations reveal an anti-correlation between the electron pressure and neutral atom density, caused by the ionization of atoms.

In this paper it is investigated how the density of neutral particles respond to a blob through a self-consistent 2D model, with dynamical plasma and neutral fields. The neutrals consist of both deuterium molecules, which are puffed into the system, and deuterium atoms originating from dissociation of the molecules. This model is explained in detail in [10]. In addition to this, a passive species of helium atoms is simulated to highlight the difference between neutral atoms that are created from volume dissociation of molecules, and those which enter the system as atoms.

It is found that densities of thermal molecules and atoms which do not originate from dissociative processes behave as reported in [7, 8], i.e., the neutral density decreases locally due to interactions with the electrons in the filament. The density of atoms from dissociated molecules, however, displays an opposite behaviour if the reaction rate for dissociation of molecules is bigger than that for ionization of atoms, which is the case in the SOL region for the results presented here. The implications for GPI calculations are that the temperature of the neutral gas, as well as the origin of the neutral atoms, dictate the amplitude, extend, and sign of the neutral density perturbation during interactions with blobs.

\section{Correlated perturbations in the scrape-off layer}

The reaction rate $R$ for electron impact reactions with neutrals have the general form

$$
R=n_{\mathrm{e}} n_{\mathrm{n}} r\left(n_{\mathrm{e}}, T_{\mathrm{e}}\right)
$$

where $n_{\mathrm{e}}$ is the electron density, $n_{\mathrm{n}}$ is the density of the neutral particles, and $r\left(n_{\mathrm{e}}, T_{\mathrm{e}}\right)$ is the reaction rate coefficient which can be parametrized as a function of electron density and temperature (see e.g. [11]). It is common practice to decompose the field into a 
mean field term and a fluctuating term, i.e.,

$$
f=\langle f\rangle+\Delta f
$$

for $f=n_{\mathrm{e}}, T_{\mathrm{e}}, n_{\mathrm{n}}$. The implications of approximating the electron density and temperature fields in the SOL region with their average value when calculating (1) are discussed in [12, 13]. Ignoring the fluctuations result in a significant difference when calculating reaction rates for blobs due to the correlation between $\Delta n_{\mathrm{e}}$ and $\Delta T_{\mathrm{e}}$. Recently, the error resulting from assuming a static neutral density when calculating emission rates have been studied as well [7, 8]. Both papers draw attention to the fact that the neutral density perturbation anti-correlates with that of the electron density, as the two are coupled trough the ionization process

$$
\mathrm{A}+\mathrm{e} \rightarrow \mathrm{A}^{+}+2 \mathrm{e}
$$

where A is an atomic species such as helium He (as for [7]) or deuterium D (as for [8]). The ionization process implies that for dynamical structures with enhanced electron pressure, such as blobs, the amount of neutrals is expected to decrease, and the amount of electrons and ions to increase, effectively anti-correlating $\Delta n_{\mathrm{n}}$ with $\Delta n_{\mathrm{e}}, \Delta T_{\mathrm{e}}$.

The importance of including molecules in addition to the atomic species for which the emission is experimentally measured is supported by the dominant interactions

$$
\begin{array}{ll}
\mathrm{D}_{2}+\mathrm{e} \rightarrow 2 \mathrm{D}+\mathrm{e}, & \mathrm{D}+\mathrm{e} \rightarrow \mathrm{D}^{+}+2 \mathrm{e}, \\
\mathrm{D}_{2}+\mathrm{e} \rightarrow \mathrm{D}_{2}^{+}+2 \mathrm{e} \rightarrow \mathrm{D}+\mathrm{D}^{+}+2 \mathrm{e}, & \mathrm{D}+\mathrm{D}^{+} \rightarrow \mathrm{D}^{+}+\mathrm{D},
\end{array}
$$

which are expressed in the deuterium atom density source term

$$
S_{\mathrm{D}}^{n}=n_{\mathrm{e}} n_{\mathrm{D}_{2}}\left[2 r_{\mathrm{dis}}\left(T_{\mathrm{e}}\right)+r_{\mathrm{m} . \mathrm{iz}}\left(T_{\mathrm{e}}\right)\right]-n_{\mathrm{e}} n_{\mathrm{D}} r_{\mathrm{iz}}\left(T_{\mathrm{e}}\right)
$$

where $n_{\mathrm{D}_{2}}$ is the density of deuterium molecules, $n_{\mathrm{D}}$ is the density of deuterium atoms, $r_{\text {dis }}$ is the molecule dissociation reaction rate, $r_{\text {m.iz }}$ is that for molecular assisted ionization, and $r_{\mathrm{iz}}$ is the ionization rate coefficient for deuterium atoms. All reaction rates are parametrized in terms of $T_{\mathrm{e}}$ according to [11]. For the interactions in (4) involvining $\mathrm{D}_{2}$ and $\mathrm{D}_{2}^{+}$, there is a finite probability that some of the resulting atoms are born in an excited state, and thus emit a D-alpha photon without a prior electron impact collision. This dissociation path is well known, see e.g. [14, and result in overestimating the deuterium atom density in GPI calculations if not accounted for. Excited atomic states are, however, not included in the model. The first term of (5) results from the molecule sink term

$$
S_{\mathrm{D}_{2}}^{n}=-n_{\mathrm{e}} n_{\mathrm{D}_{2}}\left[r_{\mathrm{dis}}\left(T_{\mathrm{e}}\right)+r_{\mathrm{m} . \mathrm{iz}}\left(T_{\mathrm{e}}\right)\right],
$$

and the second term in (5) is the electron impact ionization of atoms. For a model without molecules only the last term of (5) persists, and the neutral density source 


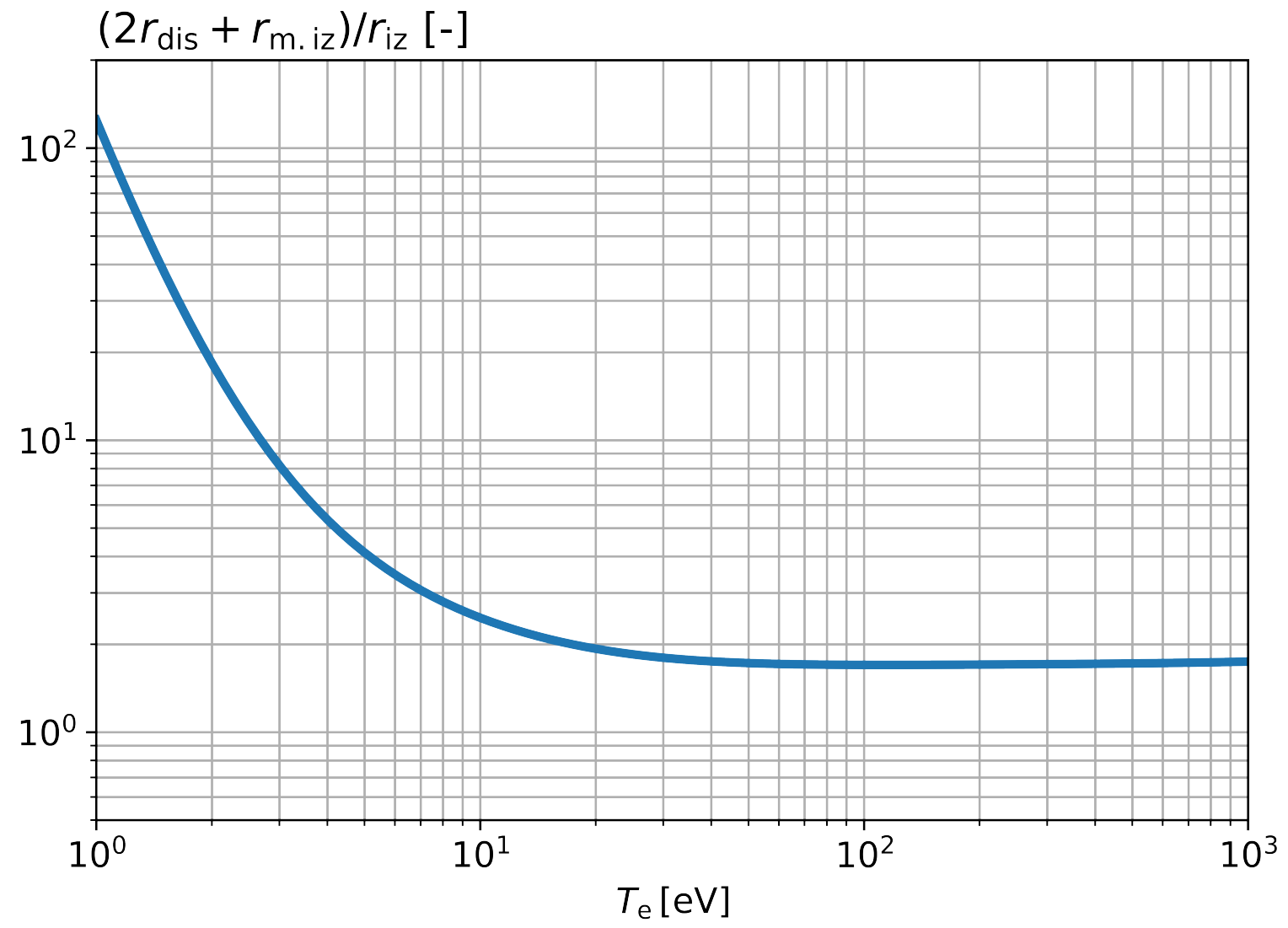

Figure 1. The ratio of the reaction rate coefficients for creation of neutral atoms by dissociation and molecular assisted ionization, to that for removal of neutral atoms by ionization, as a function of electron temperature. It is observed that for all temperatures, the ratio larger than one.

is always negative, resulting in the anti-correlation between the atom density and the electron density as observed in [7, 8]. For the source of deuterium atoms in (5), however, the sign is determined by the ratio of the absolute value of the two terms

$$
\eta=\frac{n_{\mathrm{e}} n_{\mathrm{D}_{2}}\left[2 r_{\mathrm{dis}}\left(T_{\mathrm{e}}\right)+r_{\mathrm{m} . \mathrm{iz}}\left(T_{\mathrm{e}}\right)\right]}{n_{\mathrm{e}} n_{\mathrm{D}} r_{\mathrm{iz}}\left(T_{\mathrm{e}}\right)}=\frac{n_{\mathrm{D}_{2}}}{n_{\mathrm{D}}} \frac{2 r_{\mathrm{dis}}\left(T_{\mathrm{e}}\right)+r_{\mathrm{m} . \mathrm{iz}}\left(T_{\mathrm{e}}\right)}{r_{\mathrm{iz}}\left(T_{\mathrm{e}}\right)},
$$

which dictates that the atom source term is negative for $0<\eta<1$ and positive for $\eta>1$. The last fraction in (7), i.e., the ratio of the reaction rates, is plotted in Fig. 11. It is observed that for all relevant energy scales this factor is larger than one, and increases rapidly for smaller electron energies below $10 \mathrm{eV}$. Thus, for regions where the neutral molecule density is sufficiently high, perturbations to the electron pressure result in a positive perturbation to the neutral atom density. 


\section{Numerical model}

The effect of blobs on the neutral density is investigated numerically through a combined plasma and neutral fluid model. The plasma module solves the HESEL [15, 16] fourfield drift-fluid model for the electron/ion density, electron and ion pressures, and the generalized vorticity. The neutral module solves a three-field diffusion model, which has a thermal $\mathrm{D}_{2}$ molecule species, and two more energetic atomic $\mathrm{D}$ species to include both the Franck-Condon atoms produced from dissociation and those energized by atomic charge exchange collisions with ions. The plasma and neutral modules are coupled through the source terms, and the plasma and neutrals interact self-consistently through molecular dissociation, molecular assisted ionization, atomic ionization, and atomic charge exchange collisions with ions, i.e., the interactions in (4). The combined neutral-plasma model is denoted nHESEL and is described in detail in [10].

In addition to the neutral deuterium species described in the model in [10], a passive fluid model for neutral helium gas has been added to the code. The transport equations for the helium density is identical to that of $\mathrm{D}_{2}$, as the mass and temperature of the particles are assumed to be the same, i.e.,

$$
\partial_{t} n_{\mathrm{He}}-\nabla \cdot\left(D_{\mathrm{He}} \nabla n_{\mathrm{He}}\right)=S_{\mathrm{He}}^{n}
$$

with $D_{\mathrm{He}}=D_{\mathrm{D}_{2}}$, and the source term

$$
S_{\mathrm{He}}^{n}=-n_{\mathrm{e}} n_{\mathrm{He}} r_{\mathrm{iz}, \mathrm{He}}\left(T_{\mathrm{e}}\right),
$$

and where $r_{\mathrm{iz}, \mathrm{He}}$ is the ionization reaction rate coefficient for helium in the ground state, for which the parametrization in terms of $T_{\mathrm{e}}$ is found in [11]. The source term (9) is calculated according to the dynamical helium density, and electron density and temperature fields, but information from interactions with helium does not feed back into the drift-fluid equations for the plasma fields as is the case for the other neutrals.

The full model is implemented in the BOUT ++ framework [17], and the equations are solved in a 2D slab perpendicular to the magnetic field lines at the out-board mid-plane. The domain overlaps both parts of the edge and SOL regions, and has dimensions of $225 \times 75 \rho_{\mathrm{s}}^{2}$. In the poloidal $y$-direction the boundaries are periodic, and at the inner edge boundary, the conditions are for the generalized vorticity $\omega=0$, density $n=1.5 n_{0}$, and electron and ion pressures $p_{\mathrm{e}}=2 p_{\mathrm{i}}=10 n_{0} T_{\mathrm{e} 0}$. At the outer boundary, the conditions are $\partial_{x} n=\partial_{x} p_{\mathrm{e}, \mathrm{i}}=\omega=0$, where $x$ is the radial direction. The normalization values are

$$
\begin{aligned}
\Omega_{\mathrm{ci}} & =9.57 \cdot 10^{7} \mathrm{~s}^{-1}, & \rho_{\mathrm{s}} & =3.23 \cdot 10^{-4} \mathrm{~m}, \\
n_{0} & =1.5 \cdot 10^{19} \mathrm{~m}^{-3}, & T_{\mathrm{e} 0} & =20 \mathrm{eV},
\end{aligned}
$$

which correspond to typical parameters for a medium-sized tokamak [16]. The neutral density fields for the atomic deuterium atoms $(n=$ warm, hot $)$, the deuterium molecules 


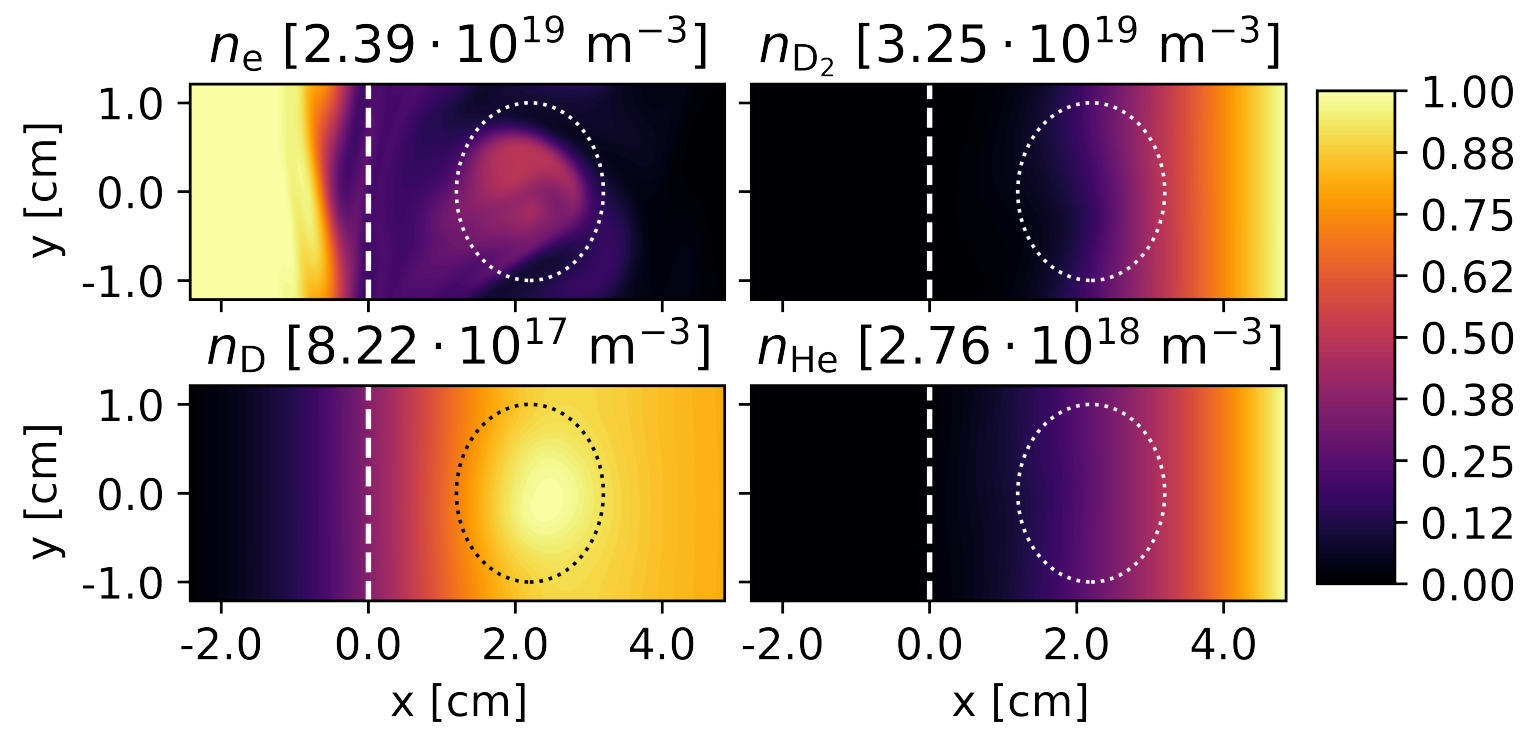

Figure 2. Normalized electron density, deuterium molecule density, deuterium atom density, and helium atom density during a blob. The blob is clearly observable from the electron density, whereas perturbations to the neutral densities are relatively smaller. The dotted straight line is the last closed flux surface, and the circle is inserted as a reference for the blob position in this and the following figures.

$\left(\mathrm{n}=\mathrm{D}_{2}\right)$, and helium atoms $(\mathrm{n}=\mathrm{He})$ satisfy

$$
-D_{\mathrm{n}} \partial_{x} n_{\mathrm{n}}=\sqrt{-\frac{D_{\mathrm{n}} S_{\mathrm{n}}^{\prime}}{n_{\mathrm{n}}}} n_{\mathrm{n}}
$$

at the inner boundary, and

$$
-D_{\mathrm{n}} \partial_{x} n_{\mathrm{n}}=-\gamma \sqrt{-\frac{D_{\mathrm{n}} S_{\mathrm{n}}^{\prime}}{n_{\mathrm{n}}}} n_{\mathrm{n}}+\Gamma_{\mathrm{n}, \text { puff }} \delta_{\mathrm{n},\left(\mathrm{D}_{2}, \mathrm{He}\right)},
$$

at the outer boundary, where $S_{\mathrm{n}}^{\prime}$ are the source terms that are proportional to $n_{\mathrm{n}}$. The choice of neutral boundary conditions is discussed in [10]. The parameters used for the neutral fields are

$$
\begin{aligned}
D_{\text {hot }} & =10^{1} D_{\text {warm }}=10^{3} D_{\mathrm{D}_{2}}=10^{2} \rho_{\mathrm{s}}^{2} \Omega_{\mathrm{ci}}, & & \Gamma_{\mathrm{D}_{2}, \text { puff }}=1 \cdot 10^{21} \mathrm{~m}^{-2} \mathrm{~s}^{-1}, \\
\gamma & =0.2, & & \Gamma_{\mathrm{He}, \text { puff }}=1 \cdot 10^{20} \mathrm{~m}^{-2} \mathrm{~s}^{-1},
\end{aligned}
$$

and the atomic deuterium density reported here is related to that of the deuterium warm and hot sub-species introduced in [10] by $n_{\mathrm{D}}=n_{\text {warm }}+n_{\text {hot }}$.

A snapshot of the densities during a blob is shown in Fig. 2. Here the atom density is the sum of the two distinct (warm and hot) deuterium atom fluid densities at different temperatures, whereas the density of helium atoms is shown separately. 


\section{Neutral response to blob}

The impact of the blob on the neutral densities is slightly visible from Fig. 2. The relative deviation from the temporal mean

$$
\delta_{\sigma}=\frac{n_{\sigma}-\left\langle n_{\sigma}\right\rangle_{t}}{\left\langle n_{\sigma}\right\rangle_{t}},
$$

for $\sigma=\mathrm{e}, \mathrm{D}, \mathrm{D}_{2}$, He is shown in Fig. 3. Here $\left\langle n_{\sigma}\right\rangle_{t}=\frac{\int \mathrm{d} t n_{\sigma}}{\int \mathrm{d} t}$ is the temporal average of the density of $\sigma$. The blob is visible in the plot for the electron density deviation $\delta_{\mathrm{e}}$ and induce a negative perturbation to the thermal neutral deuterium molecule and helium densities, and a positive perturbation to the deuterium atom density. The perturbation to the deuterium atom density is of similar amplitude to that of molecules, but the effect is visible in the whole domain. The perturbation to the molecule density is much more localized, and the trail remains visible after the blob has moved further outwards. Thermal transport time-scales are much larger for the thermal species than those for deuterium atoms. This is the reason why the the blob leaves a trail of neutral density depletion in deuterium molecule and helium atom densities, but not in the deuterium atom density. For helium atoms, the perturbation is also of smaller amplitude, as the ionization energy for helium is several times larger than the dissociation energy for deuterium molecules, and thus less helium atoms are ionized than molecules are dissociated under the same conditions.

The influence of plasma pressure perturbations, e.g. in blobs, on the neutral atom density thus depends on several factors. It is observed, that it makes a difference whether the atoms are injected directly into the system (as for He), or if they originate from volume dissociation of molecules (as for D). For the latter case, the source of atoms due to dissociation of molecules may be larger than the sink due to ionization, resulting in a positive correlation with the electron density perturbations. Whether this is the case depend on the ratio of molecules to atoms, and on the electron temperature. However, if the ratio of molecules to atoms, $n_{\mathrm{D}_{2}} / n_{\mathrm{D}}$ is above 0.58 , this will always be the case. Secondly, the temperature of the neutrals determines how local the response to the electron pressure perturbation is. If the neutrals are thermal, the equalization of a depletion or enhancement of the density is slow and the effect of the blob is more local. If the neutrals are warmer, such as those created by dissociation or charge exchange collisions, the perturbation quickly spread out to a larger volume.

The value of $\eta$ defined in (7) is shown for the whole domain in Fig. 4. It is observed that $\eta$ is larger than one in most of the SOL, and that the blob brings this value closer to unity as expected from Fig. 1, and because increased plasma density remove molecules and create atoms. Note that the value of the source ratio $\eta$ only determines the sign, and not the amplitude, of the perturbation. Thus, blobs pushing $\eta$ closer to unity still 


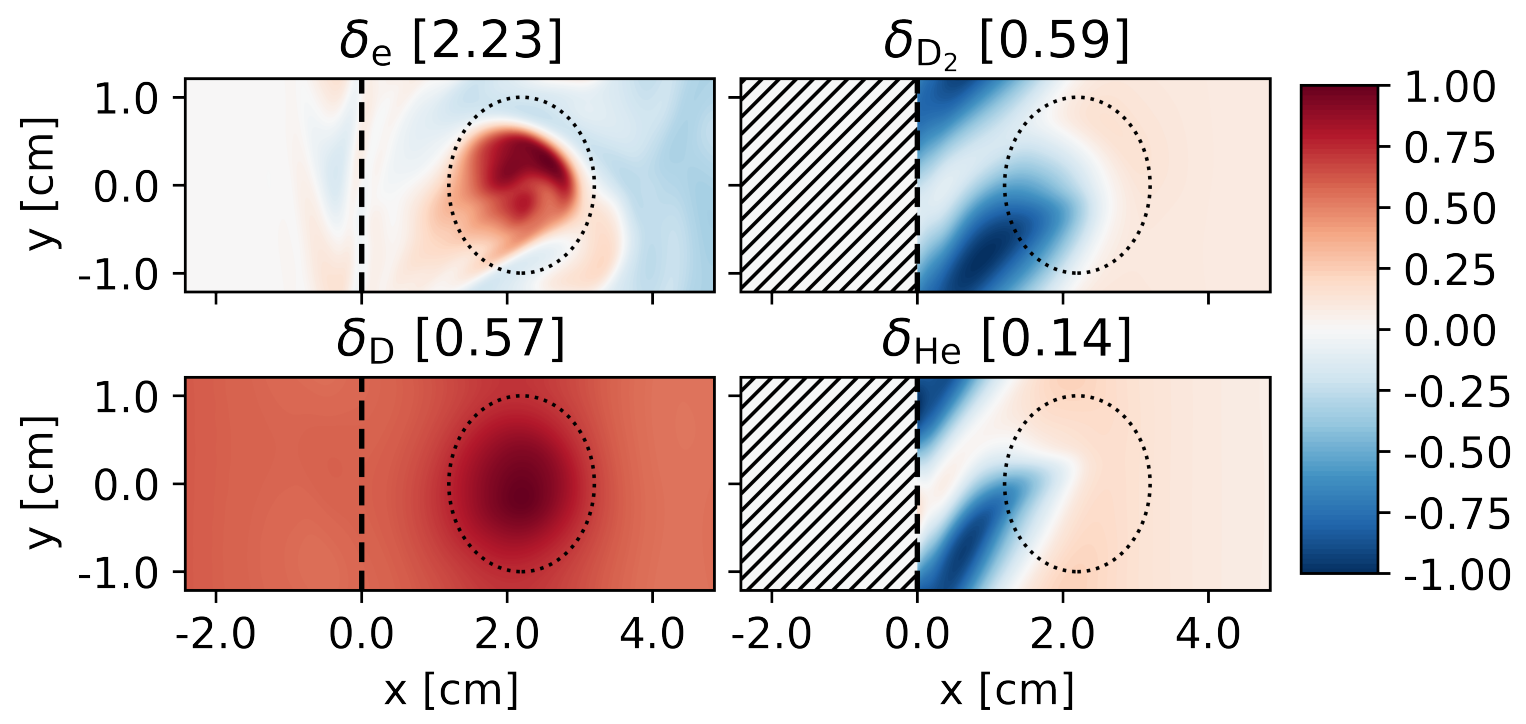

Figure 3. Deviation of the electron and neutral densities from their temporal mean values. The blob result in a negative perturbation in the thermal deuterium molecule and helium atom densities, and a positive perturbation in the deuterium atom density. The data for the thermal neutrals in the shaded areas are excluded, as the neutral density here is so low that the relative fluctuations outshine those resulting from the blob interactions.

result in an enhanced perturbation, since the magnitude of the sources increase with the blob pressure. Although only the effects of a single blob created from one set of plasma and neutral input parameters are studied, the results are expected to reproduce well in the range of realistic SOL pressures. At lower temperatures, less molecules are removed, and the ratio of the reaction rate coefficients shown in Fig. 1, increases drastically, which increases $\eta$ and enhance the positive response of the neutral density to blobs reported here. Higher pressures may result in a lower $\eta$. At higher temperatures, the ratio of the reaction rate coefficients in Fig. 1 retains a value of approximately 1.7, but a decrease in the density of $\mathrm{D}_{2}$ as a result of enhanced electron pressure, causes the value of $\eta$ to decrease as well. This is the case in the edge region in Fig. 4. For higher edge pressures blobs drag the sub-one region of $\eta$ further into the SOL, as a result of the increased depletion of $\mathrm{D}_{2}$.

Opposed to the temperature dependent part of $\eta$, which is shown in Fig. 1, it is difficult to make a generic estimate of the ratio of the density of $\mathrm{D}_{2}$ to that of $\mathrm{D}$. In experiments the densities depend on both neutral and plasma variables, as well as the geometry of the machine and on the first wall and divertor materials. A simple 0D model may, however, elucidate the temporal characteristics of the ratio and provide a characteristic time-scale for the change of the density ratio compared to the time-scale set by blob propagation. Consider a neutral model for the densities of $\mathrm{D}_{2}$ and $\mathrm{D}$ similar to that presented previously, but with the temporal evolution entirely determined by the source 


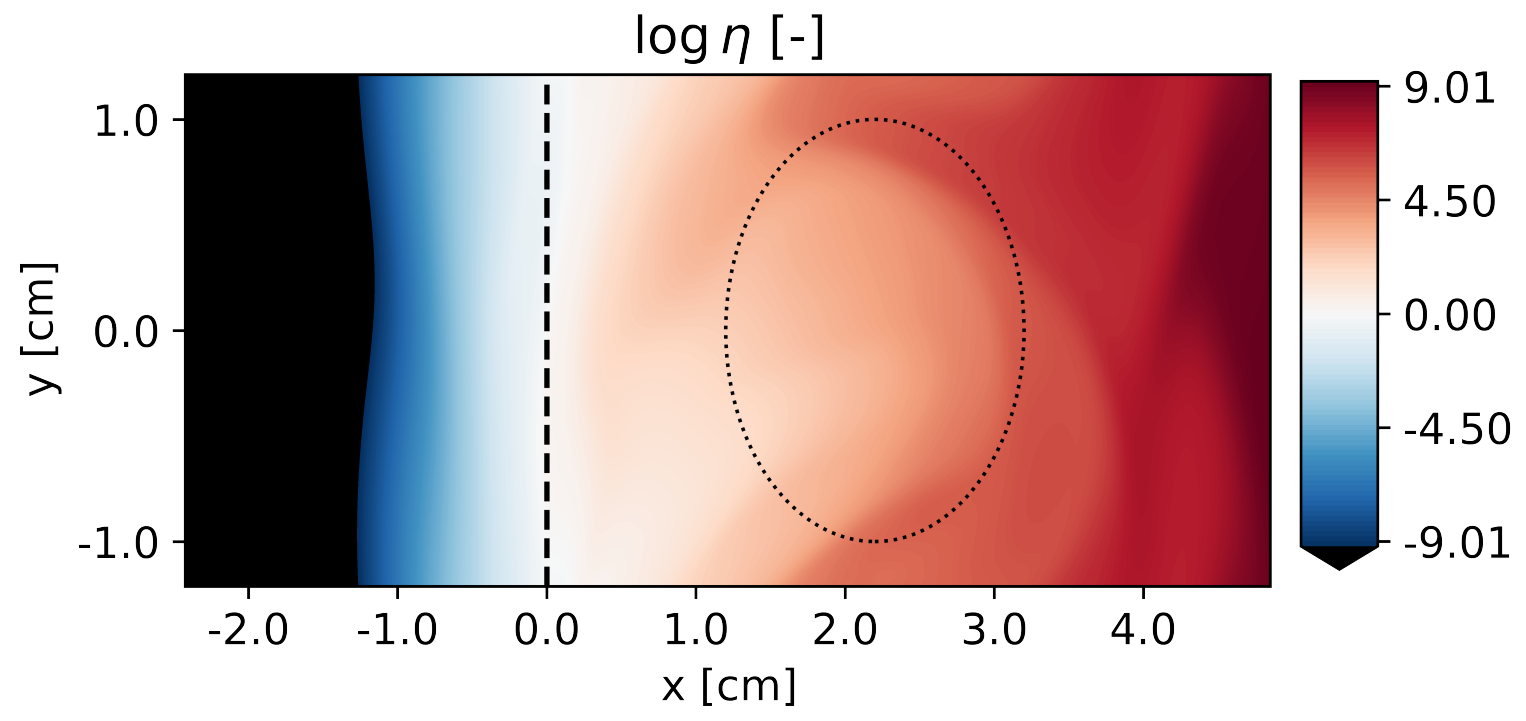

Figure 4. The logarithm of the ratio of creation of neutral atoms to that of ionization of neutral atoms $\eta$, defined in Eqn. (7). The ratio is larger than one in most of the SOL region.

terms, i.e.,

$$
\partial_{t} n_{\mathrm{n}}=S_{\mathrm{n}}^{n}
$$

where $\mathrm{n}=\mathrm{D}_{2}, \mathrm{D}$, and $S_{\mathrm{D}_{2}}^{n}$ and $S_{\mathrm{D}}^{n}$ are those in (5) and (6). Assuming a constant electron density and temperature, and initial values of the densities of $n_{\mathrm{D}_{2}}=n_{\mathrm{D}}=n_{0}$, an analytical solution is readily obtained and reads

$$
\begin{aligned}
n_{\mathrm{D}_{2}} & =n_{0} \mathrm{e}^{-n_{\mathrm{e}}\left(r_{\text {dis }}+r_{\text {m.iz }}\right) t}, \\
n_{\mathrm{D}} & =n_{0} \frac{\mathrm{e}^{-n_{\mathrm{e}} r_{\mathrm{iz}} t}\left(\left(2 r_{\text {dis }}+r_{\text {m.iz }}\right) \mathrm{e}^{n_{\mathrm{e}}\left(r_{\mathrm{iz}}-r_{\text {dis }}-r_{\text {m.iz }}\right) t}+r_{\text {iz }}-3 r_{\text {dis }}-2 r_{\text {m.iz }}\right)}{r_{\text {iz }}-r_{\text {dis }}-r_{\text {m.iz }}} .
\end{aligned}
$$

The solutions are an exponentially decaying molecule density, whereas the atom density has terms of both growth and decay, consistent with the more elaborate 2D model. For typical SOL parameters the solutions are shown in Fig. 5, and an initial growth of the neutral atom density followed by an exponential decay when the neutral molecule density depletes. The analytical solution provides a characteristic time-scale for the decay of the density ratio $n_{\mathrm{D}_{2}} / n_{\mathrm{D}}$, which can be compared to the time-scale set by blob dynamics. The ratio drops from its initial value of 1 to $\frac{1}{2}$ after approximately $50 \mu \mathrm{s}$. The time it takes for a blob to move a distance corresponding to its width of approximately $1 \mathrm{~cm}$ at a typical speed of $1 \mathrm{~km} / \mathrm{s}$ is $10 \mu \mathrm{s}$. Thus, a blob can only change the ratio locally by a fraction before it has passed. This observation suggests that a general estimate of the neutral density ratio, and thus of $\eta$ and the sign of the perturbation to the deuterium density, is accessible from the average effect of blobs or from an assumed static background plasma, and can thus be obtained from more experiment-specific 


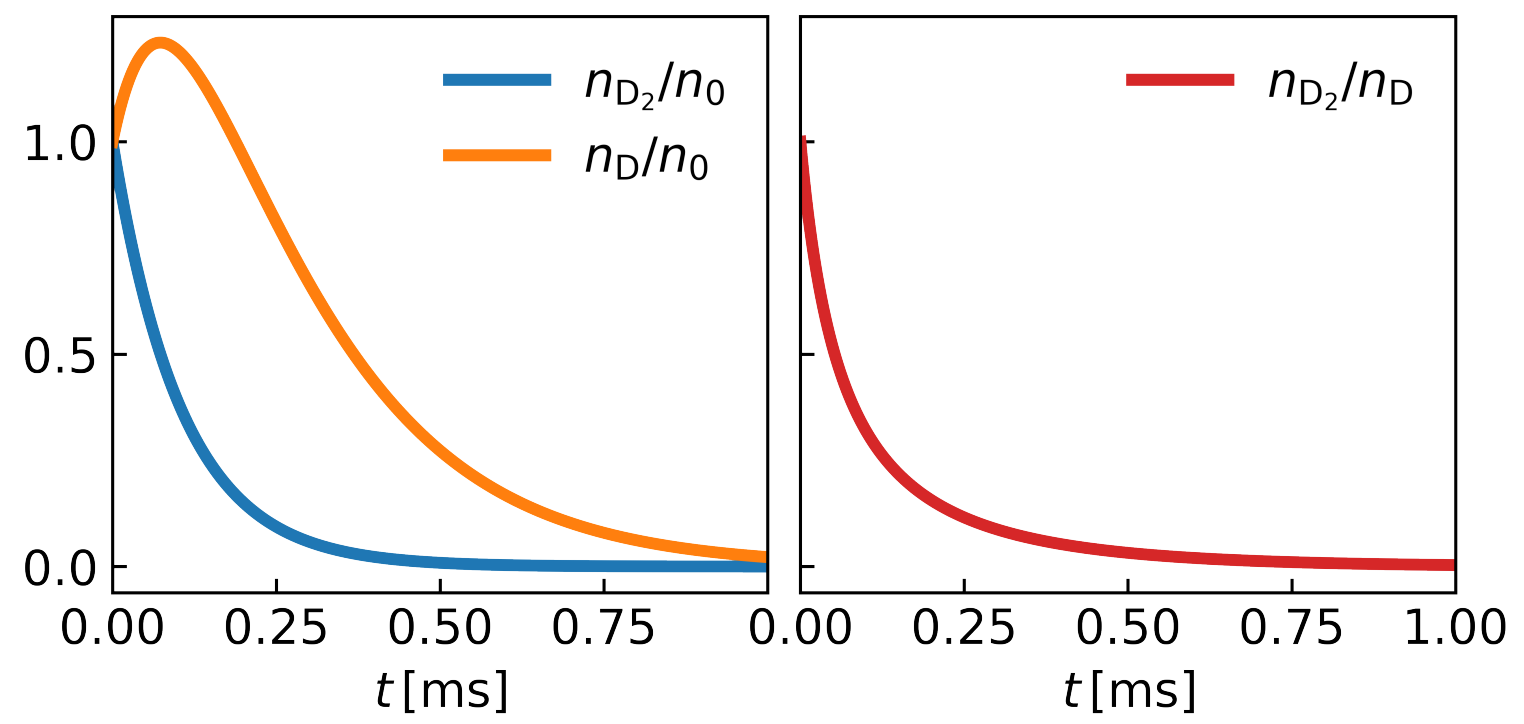

Figure 5. The solution for the simplified $0 \mathrm{D}$ neutral model, with $n_{0}=n_{\mathrm{e}}=10^{18} \mathrm{~m}^{-3}$ and $T_{\mathrm{e}}=10 \mathrm{eV}$, is shown in the plot to the left. It is observed that the deuterium atom density initially increases due to the dissociation of molecules, and falls off exponentially when the molecular density is depleted. The corresponding ratio of $\mathrm{D}_{2}$ to $\mathrm{D}$ is shown in the plot to the right and provides a characteristic time-scale for the change of the density ratio.

neutral codes, that do not necessarily involve a dynamical treatment of plasma and neutral fields.

In addition to the study of the specific case presented here, the value of $\eta$ is calculated for several simulations with different levels of gas-puffing, and thus a wide span of neutral densities. The results are shown in Fig. 6, and allow for a more generic calculation of $\eta$ than can be obtained from studying a single blob event. For the simulations considered it is observed that $\eta$ display the same strong radial dependence as in Fig. 4 . This is due to the difference in the length scales of transport of molecules and atoms caused by the large difference in their temperature. It is also observed that the value of $\eta$ is generally above 1 outside the LCFS, and a positive perturbation to the atom density caused by blobs can thus be expected in the whole SOL region.

Both the simple 0D model for the density ratio and the comparison of the radial dependence of $\eta$ for multiple simulation support the conclusion. Blobs locally affect the value of $\eta$, but it only changes around the value determined by a background or statistically steady state plasma. This allows for applying numerical steady state neutral models that take many variables into account to estimate in which regions $\eta$ is generally above or below the threshold value of 1 , and thus the expected sign of the atomic perturbation during blobs. The amplitude of the perturbation, however, cannot be calculated from the average profiles, and requires support from a dynamical code such 

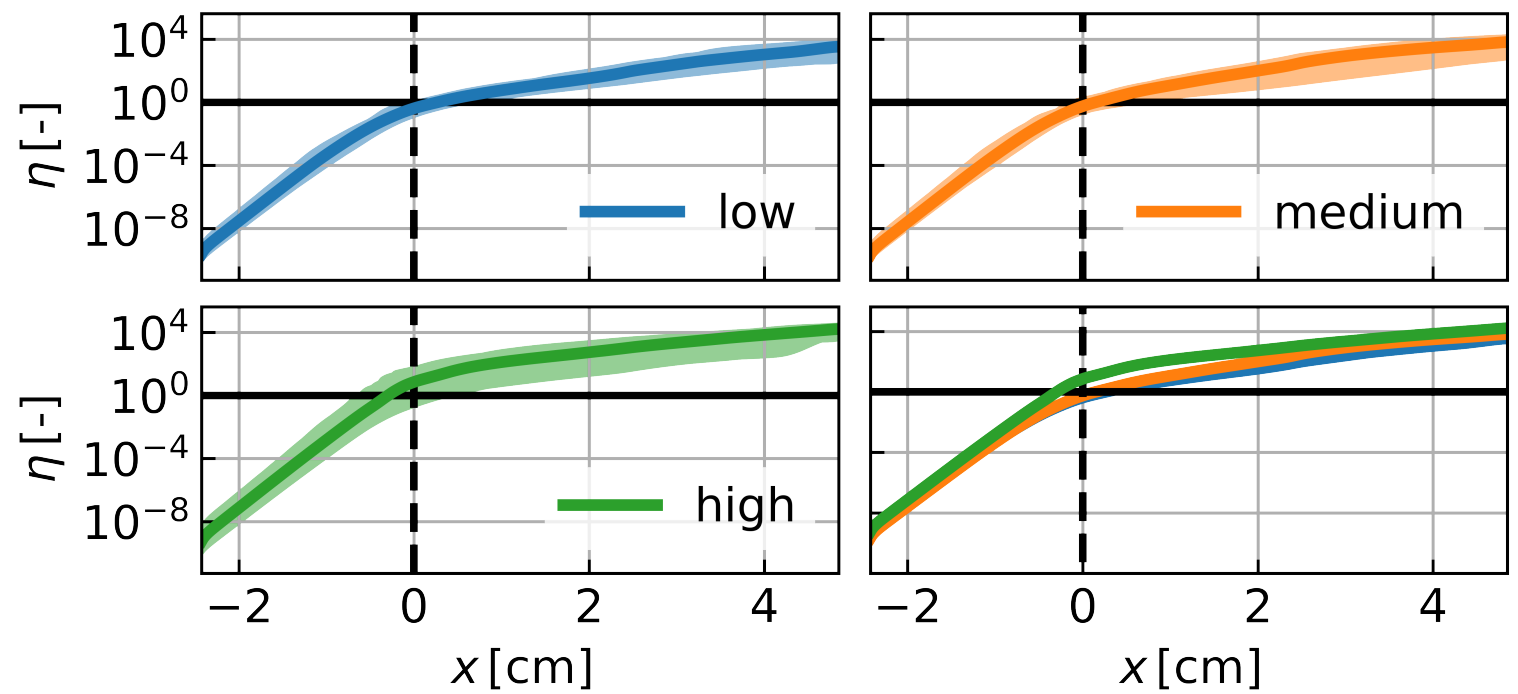

Figure 6. The solid lines show the poloidally and temporally averaged value of $\eta$ for three simulations with low, medium, and high gas puffing respectively, similar to those presented in [10. The shaded regions surrounding the solid lines cover the values of $\eta$ for all poloidal and temporal instances at a given radial position, and thus indicate the spread in $\eta$ caused by the intermittency of the plasma and neutral fields. In the lower right plot the average values for $\eta$ are overplotted. It is observed that the value of $\eta$ generally increases with the puffing rate, most likely due the resulting enhanced cooling of the electrons. The vertical dashed line indicate the radial position of the LCFS, and the horizontal solid line shows $\eta=1$. It is observed that the value of $\eta$ generally is above 1 in the SOL region, and increases exponentially for larger radial positions.

as nHESEL.

The simulated thermal helium density shows a behaviour similar to that observed for neutral atoms in [7, 8], and thus the conclusion on the effect of assuming a constant neutral background in calculations based on GPI measurements is the same. That is, the neutral atoms anti-correlate with the electron pressure perturbations, and when calculating reaction rates of the form in (1) the product is therefore overestimated if a static neutral density is assumed. The results for the density of non-thermal neutral atoms that originate from dissociation of molecules, however, may have a much lower, or even the opposite, effect for GPI if this is based on radiation of such atoms, which for example is the case for atomic D-alpha measurements. 


\section{Conclusions}

The effect of electron pressure perturbations mediated in blobs on the densities of neutral atoms and molecules in the SOL is investigated by a numerical model. The densities of the neutrals and of the electrons are strongly coupled through in-elastic sources, and perturbations in the electron pressure result in an immediate perturbation to the neutral density.

The neutral density response to blobs is investigated by a coupled neutral-plasma model, that self-consistently describe the interplay between neutral densities, and the turbulent density and pressure fields of electrons and ions. The neutral part of the model describes the evolution of deuterium atom and molecule densities, as well as that of a passive helium species.

For molecules, the blob induced perturbation anti-correlates with that for electron density, which result in increased magnitude of the molecule density sink terms. The same is the case for atoms, that do not originate from dissociation of molecules (e.g. helium atoms). However, for atoms that are created from dissociative processes the density perturbation can correlate with that of the electron pressure, as more atoms are created from dissociation of molecules than removed by ionization.

The response of the atom densities to blobs is important when interpreting atomic emission, e.g., GPI measurements, since assuming a constant neutral background will either lead to over- or under-estimating the amplitude of the electron pressure perturbation, depending on the SOL conditions and on the origin of neutrals. Thus, when correcting for the neutral density mean-field approximation, one should also take those parameters into account. Neglecting the atom particle source from dissociation of molecules only affects the interpreted amplitude of the measured signal, and not properties derived from GPI measurements such as the blob propagation velocity. There is, however, no generic recipe for how to estimate the error made by neglecting the dissociation source. The blob event presented here introduces an error of approximately $50 \%$ at the centre of the perturbation, when the blob is in the middle of the SOL region, but the value depends on several parameters. There is in particular a strong dependence on the $n_{\mathrm{D}_{2}} / n_{\mathrm{D}}$ ratio, which as discussed in Sec. 4 is difficult to determine in general. Thus, for practical applications it is advised to estimate the value of $\eta$, e.g., from simulations with kinetic models such as EIRENE [18], which will reveal whether, and in which regions, $\eta$ diverges significantly from unity. For more quantitative assessments a dynamical model such as nHESEL can subsequently be applied to quantify neutral density perturbations. 


\section{Acknowledgements}

The authors thank Eduardo de la Cal for insightful discussions on neutral density perturbations and sources of D-alpha radiation.

This work has been carried out within the framework of the EUROfusion Consortium and has received funding from the Euratom research and training programme 2014-2018 under grant agreement No 633053. The views and opinions expressed herein do not necessarily reflect those of the European Commission.

\section{References}

[1] A. Loarte, et al., Progress in the ITER Physics Basis Chapter 4: Power and particle control 2007 Nucl. Fusion 47 S203

[2] S.J. Zweben, et al., Plasma Phys. Control. Fusion 49, S1 (2007)

[3] D.A. DIppolito et al., Physics of Plasmas 18, 060501 (2011)

[4] J.L. Terry, et al., J. Nucl. Mater. 290 757, (2001)

[5] S.J. Zweben, et al., Physics of Plasmas 9 1981, (2002)

[6] R.J. Maqueda, et al., Review of Scientific Instruments 74 2020, (2003)

[7] E. de la Cal and The TJ-II Team, Nucl. Fusion 56, (2016) 106031

[8] C. Wersal and P. Ricci, Nucl. Fusion 57, (2017) 116018

[9] D. Moulton, et al., J. Nucl. Mater. 463 893, (2015)

[10] A. Thrysøe, et al., Physics of Plasmas 25, 032307 (2018)

[11] R.K. Janev, et al., Elementary Processes in Hydrogen-Helium Plasmas, Berlin: Springer-Verlag, (1987)

[12] E. Havlíčková, et al., J. Nucl. Mater. 415 (2011) S4714

[13] A. Thrysøe, et al., Plasma Phys. Control. Fusion 58, (2016) 044010

[14] E. de la Cal, et al., Nucl. Fusion 48, (2008) 095005

[15] J. Madsen, et al., Physics of Plasmas 23, 032306 (2016)

[16] A.H. Nielsen, et al., Plasma Phys. Control. Fusion 59, 025012 (2017)

[17] B.D. Dudson, et al., Computer Physics Communications 180 (2009), pp. 1467-1480

[18] D. Reiter, et al., Fusion Science and Technology, 47, 172 (2005) 